\title{
Gasto Privado en Salud de los Hogares de Cartagena de Indias, 2004
}

\author{
Household spending on private health care in \\ Cartagena de Indias, 2004
}

\author{
Luís Alvis-Estrada ${ }^{1}$, Nelson Alvis-Guzmán ${ }^{1}$ y Fernando de la Hoz ${ }^{2}$ \\ 1. Departamento de Investigaciones Económicas y Sociales. Universidad de Cartagena, Colombia. \\ lalvis20@yahoo.com, nalvis@yahoo.com \\ 2. Departamento de Salud Pública, Facultad de Medicina. Universidad Nacional de Colombia. \\ fpdelahozr@unal.edu.co
}

Recibido 4 Mayo 2006/Enviado para Modificación 8 Octubre 2006/Aceptado 12 Enero 2007

\section{RESUMEN}

Objetivo Estimar el gasto privado en salud (gasto de bolsillo) de los hogares de Cartagena de Indias y su correlación con características del jefe del hogar (estrato socioeconómico al cual pertenece, escolaridad, afiliación al Sistema General de Seguridad Social en Salud y vinculación laboral).

Métodos Estudio transversal sobre una muestra de 384 hogares establecida mediante muestreo estratificado proporcional a los estratos socioeconómicos. Los datos fueron recolectados mediante formulario estandarizado, tabulados y procesados utilizando el paquete estadístico SPSS $® 10.0$ para estimar los modelos econométricos.

Resultados Los estratos más bajos (1 y 2) incurren en un gasto de bolsillo mayor con relación a su ingreso, que los estratos más altos. Un bajo nivel de escolaridad de los jefes de hogar, la condición de desempleado o trabajador independiente, y el hecho de no tener afiliación al SGSSS implican un mayor porcentaje de gasto de bolsillo en salud en proporción a su ingreso.

Conclusiones La expansión de los mecanismos del mercado en los servicios de salud, al interior del sistema de salud colombiano, ha hecho que una de las modalidades de financiamiento (gasto de bolsillo) profundice la inequidad en el financiamiento. La experiencia demuestra que esta modalidad de financiamiento se ha transformado en una barrera para el acceso a los servicios de salud.

Palabras Clave: Sistemas de salud, financiación, equidad, gasto en salud. Cartagena de Indias (fuente: DeCS, BIREME).

\section{ABSTRACT}

Objective Determining household out-of-pocket payments for access to health care services in Cartagena, Colombia, and its correlation with head of household characteristics (socioeconomic level, schooling, general social health security system (GSHSS) affiliation and type of work).

Methods A cross-sectional study was carried out on a sample of 384 homes selected by 
using proportional stratified random sampling. Data was collected using a standardised form. SPSS (version 10.0) was used for statistical and econometric analysis.

Results The lowest socioeconomic strata (1 and 2) were incurring higher out-of-pocket expenditure than higher strata (3-6). Other factors predicting greater expenditure on health compared to income were household heads' low level of schooling, being unemployed or working independently and the fact of not being affiliated to the GSHSS. Conclusions Market mechanism expansion into the Colombian health system has resulted in greater inequality in terms of out-of-pocket payment for the lowest social strata. Our findings suggest that the need for such financing represents an important barrier for gaining access to health care.

Key Words: Health system, financing, equity, out-of-pocket expenditure (source: MeSH, $N L M)$

$\mathrm{E}$

l gasto en salud se reconoce como una inversión en capital humano ya que permite que las personas alcancen una calidad de vida adecuada y contribuye al crecimiento económico de las naciones (1). Sin embargo, el gasto privado (de bolsillo) es la forma más inequitativa de financiar servicios de salud, porque la atención médica está en función de la capacidad de pago y no de las necesidades de la salud de las familias, ya que al momento de enfermar una persona, no podrá negociar efectivamente el precio del servicio que recibirá, ni elegir el de mejor calidad (2).

El modelo de Grossman, es considerado por muchos, como la innovación teórica más importante en economía de la salud de los últimos años (3). En éste, la salud se considera un stock de capital duradero cuyo producto es tiempo sano, el cual puede ser utilizado para la obtención de rentas salariales o monetarias en el mercado y para la producción de "mercancías" en el hogar o sector de no-mercado. Por tanto, el individuo lo que demanda es tiempo sano -por ejemplo, tantos días sanos al año- por su utilidad directa para la producción de mercancías y por su utilidad como inversión para conseguir mayores rentas monetarias: no porque se suponga que el individuo con mayor salud puede obtener un mayor salario, lo cual no ocurre, según este modelo, sino porque no pierde días de trabajo como consecuencia de la enfermedad. Según Grossman (3) la demanda de cuidados médicos está positivamente correlacionada con el salario, porque cuanto mayor es éste: i) mayor es el menoscabo en la renta como consecuencia de los días perdidos por enfermedad y ii) mayor es el costo de oportunidad del tiempo dedicado a producir salud con otros inputs alternativos al sistema sanitario: por ejemplo durmiendo o descansando más.

En recientes estudios, como el realizado por Torres y Knaul se analizó el 
gasto de bolsillo en salud en México y los principales factores asociados que conducen a que dicho gasto sea catastrófico para las familias (mayor a $30 \%$ del gasto total menos el gasto en alimentos) (2). Entre los resultados, se encuentra el hecho de haber identificado el acceso a la seguridad social como un determinante significativo que se relaciona de forma negativa con el hecho de que las familias financien sus necesidades de salud de su propio bolsillo (2).

En Colombia, el Grupo Cendex (4) analizó el uso y gasto en salud a través de un monitoreo de cerca de 6000 familias durante más de un año en cuatro ciudades. Ellos encontraron una asociación entre el gasto de bolsillo y variables como: el ingreso familiar, el tipo de afiliación al sistema de seguridad social y tipo de servicios utilizados. En forma similar, el presente estudio fue realizado en la ciudad de Cartagena de Indias con el objetivo de medir el gasto de bolsillo en salud en las familias y observar sus relaciones con el grado de escolaridad del jefe del hogar, su vinculación a la actividad laboral, su afiliación al Sistema General de Seguridad Social en Salud- SGSSS y estrato socioeconómico al que pertenece el hogar.

El análisis de las fuentes de financiamiento y del gasto del sector de la salud es un tema relevante por su impacto no solo en la sostenibilidad financiera de los sistemas de salud, sino también en el acceso que efectivamente la población puede lograr. El análisis de la composición de las fuentes de financiamiento y su comparación con el acceso diferencial a los servicios de salud por los distintos grupos de población permite extraer conclusiones sobre el grado de equidad financiera del sistema (5).

\section{MATERIALES Y MÉTODOS}

Se llevó a cabo un estudio transversal, con el propósito de medir el gasto de bolsillo en salud en las familias de una comunidad específica (hogares de la ciudad de Cartagena de Indias-Colombia), y además, establecer sus relaciones con el grado de escolaridad del jefe del hogar, su vinculación a la actividad laboral, su vinculación a la actividad laboral, su afiliación al SGSSS y estrato socioeconómico al que pertenece el hogar.

\section{Población y muestra}

El universo estuvo integrado por los $276255^{1}$ hogares de Cartagena de Indias distribuidos por estrato socioeconómico. Se definió una muestra de 384 hogares fijando un nivel de confianza del 95 \% y un error máximo esperado de $5 \%$. 
Dada la heterogeneidad de la distribución de los hogares, se utilizó un muestreo estratificado proporcional a la distribución de hogares según la estratificación socioeconómica de la ciudad establecida por la Secretaría de Planeación Distrital. La Tabla 1 muestra la fracción de muestreo y el número de hogares a encuestar para cada uno de los estratos socioeconómicos de Cartagena.

Tabla 1. Distribución de los hogares muestreados por Estrato socioeconómico. Cartagena de indias 2004

\begin{tabular}{cccc}
\hline Estrato & $\begin{array}{c}\text { Total } \\
\text { hogares }\end{array}$ & $\begin{array}{c}\text { Fracción } \\
\text { de muestreo (\%) }\end{array}$ & $\begin{array}{c}\text { Hogares a } \\
\text { encuestar }\end{array}$ \\
\hline 1 & 69064 & 250 & 96 \\
2 & 85639 & 310 & 119 \\
3 & 71826 & 260 & 100 \\
4 & 22100 & 80 & 31 \\
5 & 13823 & 50 & 19 \\
6 & 13803 & 50 & 19 \\
\hline TOTAL & 276255 & 1000 & 384 \\
\hline
\end{tabular}

Recolección y análisis de datos

Los datos fueron recolectados utilizando un formulario estandarizado y aplicado por estudiantes de último semestre del programa de Economía de la Universidad de Cartagena a los jefes de los hogares seleccionados en la muestra, en diversas instituciones prestadoras de servicios de salud de Cartagena de Indias, durante el segundo semestre del año 2004. En dicho formulario se integró información sobre el nivel de ingreso de los hogares, el gasto de bolsillo en salud y sus características socioeconómicas y demográficas. El gasto de bolsillo en salud se desagregó según los distintos servicios de salud, tales como, pago de seguro medico privado, consulta odontológica, consulta médica, hospitalización y cirugía, medicamentos, y otros servicios. Para las variables de gasto e ingreso, se tuvo en cuenta la información del último trimestre. La información recolectada fue integrada a una base de datos y analizada utilizando el paquetes estadístico SPSS ${ }^{\circledR}$ 10.0. Para valorar la relación entre condición laboral y afiliación a la seguridad social en salud, se utilizó la prueba Ji cuadrado. Y, dado que el estadístico Ji Cuadrado solo indica la existencia o no de relación de asociación entre dos variables, pero no el grado de ésta, se procedió a determinarlo a través de una medida direccional para variables ordinales como la "d de Somer" (6). Para valorar la incidencia de la escolaridad sobre el gasto 
se realizó una diferencia de medias de los porcentajes de gasto privado, entre los niveles más bajos y los más altos de escolaridad.

Modelo de análisis

Con el objetivo de determinar si las variables planteadas explican o no el gasto de bolsillo en salud se desarrolló el siguiente modelo:

$$
G_{h}=\alpha_{0}+\alpha_{1} * E S_{j}+\alpha_{2} * E_{j}+\alpha_{3} * A_{j}+\alpha_{4} E S T_{j}+\mu_{j}
$$

Donde: $\alpha_{k}<0 ; k=1,2,3,4$

$\mu \mathrm{j}$ es el término de error con una media igual a cero y varianza constante. Los $\alpha 1, \alpha 2, \alpha 3$ y $\alpha 4$ son los coeficientes desconocidos que serán estimados. Gh es el gasto privado en salud (gasto de bolsillo) en proporción del ingreso del hogar h; ESj es el nivel de escolaridad del jefe del hogar (sin escolaridad, primaria incompleta, primaria completa, secundaria incompleta, secundaria completa, técnico, universitario, postgrado), Ej es la condición laboral (desempleado, trabajador independiente, jubilado y trabajador dependiente); ESTj es el estrato socioeconómico donde residía el jefe de hogar (del 1 al 6) y Aj es el tipo de afiliación a la seguridad social (sin afiliación, régimen subsidiado y régimen contributivo)

Para estimar las variables que explican el aumento o disminución de la probabilidad que el hogar incurra en gastos de bolsillo superiores a ciertos parámetros (30 \% y $50 \%$ ), se desarrolló un modelo logístico multivariado de elección binaria (6), más apropiados para estudios observacionales, como éste, en los cuales se listan las categorías de las variables independientes reportando razones de ventaja (odds ratios) con relación a la primera categoría. La OMS (7) define que un gasto en salud mayor del $50 \%$ del ingreso corriente del hogar es un gasto catastrófico. Para países en vía de desarrollo como Colombia, este porcentaje se ubica en el 30\% (8). En este modelo la variable dependiente es dicotómica $\mathrm{Y}=1$, si el gasto de bolsillo en salud es mayor o igual al $30 \%$ o $50 \%$ del ingreso del hogar; $\mathrm{Y}=0$, si el gasto de bolsillo en salud es menor al $30 \%$ o $50 \%$ del ingreso del hogar. 


\section{RESULTADOS}

\section{Condición laboral y afiliación al SGSSS}

Respecto la afiliación de los 384 jefes de hogar encuestados al SGSSS, discriminado según condición laboral, en los desempleados cerca del 45 \% se encuentran afiliados al régimen subsidiado y casi un tercio (30 \%) no posee ningún tipo de afiliación lo que los coloca en una situación de vulnerabilidad e incapacidad a la hora de demandar servicios médicos. Hay que aclarar que solo cuentan con el carné de SISBEN que les rebaja los gastos de los servicios médicos demandados. Un $25 \%$ de los desempleados aparecen en el régimen contributivo en calidad de beneficiarios. En cuanto a los jubilados casi el total (99 \%) se encuentran afiliados al régimen contributivo, como lo establece la norma. El $1 \%$ restante se debe a un caso de doble afiliación, una persona que estando afiliada a un régimen exceptuado (fuerzas militares), se encontró afiliada al Régimen Subsidiado. En el grupo de los trabajadores asalariados cerca del $90 \%$ aparecen en el régimen contributivo, lo que muestra una evasión cercana al $10 \%$. Hay un $4 \%$ de los asalariados que se encuentran en el régimen subsidiado. De los trabajadores independientes el $42 \%$ no tienen ninguna afiliación, eso implica que corren con todos los gastos en salud. Un tercio de los trabajadores independientes cotizan a la seguridad social, mientras que un cuarto de ellos se encuentran en el régimen subsidiado.

El nivel de afiliación del jefe de hogar afecta el conjunto de posibilidades de elección y por lo tanto el gasto privado en salud como proporción del ingreso. Hemos visto como la afiliación está asociada con el nivel de escolaridad y la condición laboral. Los resultados muestran que al cambiar el nivel de afiliación, es decir, pasar de no tener afiliación a poseer un carné de ARS ó EPS, disminuye el gasto privado (de bolsillo) en salud como proporción del ingreso. Además se hallaron diferencias significativas en cuanto a la participación del gasto privado en salud, entre los que no tienen ninguna afiliación y los del régimen contributivo $(p=0,00)$, mientras que no existe diferencia entre los no afiliados y los afiliados al régimen subsidiado $(p=0,073)$.

La condición laboral y afiliación a la seguridad social en salud reveló una asociación $(\mathrm{p}<0,05)$, y una relación positiva pero no tan fuerte $(\mathrm{d}$ de Somer $=$ 0,469). Además, encontramos que a medida que aumenta la escala de la condición laboral, disminuye la participación del gasto privado en salud en el ingreso de los hogares, Tabla 2. 
Tabla 2. Proporción del gasto trimestral en salud sobre el ingreso trimestral de los hogares, según condición laboral de los jefes de hogar encuestados. Cartagena de Indias 2004

\begin{tabular}{lccc}
\hline \multicolumn{1}{c}{ Ocupación } & $\begin{array}{c}\text { Promedio de gasto } \\
\text { en salud (trimestral) }\end{array}$ & $\begin{array}{c}\text { Ingreso corriente } \\
\text { (trimestral) }\end{array}$ & $\begin{array}{c}\% \text { del } \\
\text { gasto }\end{array}$ \\
\hline Desempleado & 334981 & 877778 & 38,1 \\
$\begin{array}{l}\text { Trabajador } \\
\text { independiente }\end{array}$ & 343834 & 1387912 & 24,7 \\
Jubilado & 585715 & 2847945 & 20,5 \\
Trabajador asalariado & 456897 & 2452941 & 18,6 \\
\hline \multicolumn{1}{c}{ Total general } & 418792 & 1912500 & 21,9 \\
\hline
\end{tabular}

Tabla 3. Proporción del gasto privado trimestral en salud en el ingreso trimestral.

Cartagena de Indias 2004

\begin{tabular}{lrccc}
\hline \multicolumn{1}{c}{ Variables } & N & $\begin{array}{c}\text { Media de gasto } \\
\text { Trimestral }\end{array}$ & $\begin{array}{c}\text { Media de ingreso } \\
\text { trimestral }\end{array}$ & \% del gasto \\
\hline Estrato Socioeconómico & & & & \\
Estrato SE (1) & 96 & 271026 & 706250 & 38,38 \\
Estrato SE (2) & 119 & 411130 & 1318487 & 31,18 \\
Estrato SE (3) & 100 & 311893 & 2448000 & 12,74 \\
Estrato SE (4) & 31 & 742200 & 3512903 & 21,13 \\
Estrato SE (5) & 19 & 786895 & 3742105 & 21,03 \\
Estrato SE (6) & 19 & 897263 & 4468421 & 20,08 \\
\hline Total & & 418792 & 1912500 & 21,90 \\
\hline Afiliación al SGSSS & & & & \\
Sin Afiliación & 92 & 339222 & 939130 & 36,1 \\
\hline Afil. Régimen Subsidiado & 59 & 158966 & 594915 & 26,7 \\
Afil. Régimen Contributivo & 233 & 509913 & 2630472 & 19,3 \\
\hline Escolaridad del jefe de hogar & & & & \\
Sin Escolaridad & 11 & 100727 & 572727 & 17,5 \\
\hline Primaria Incompleta & 23 & 302239 & 952173 & 31,7 \\
\hline Primaria completa & 65 & 478869 & 1140000 & 42,0 \\
\hline Secundaria Incompleta & 64 & 285351 & 1368750 & 20,8 \\
\hline Secundaria completa & 79 & 412734 & 1705063 & 24,2 \\
\hline Técnico & 71 & 455533 & 2395774 & 19,0 \\
\hline Universitario & 59 & 466659 & 3198305 & 14,5 \\
Postgrado & 12 & 788916 & 4250000 & 18,5 \\
\hline
\end{tabular}

Estrato socioeconómico y gasto de bolsillo

Los estratos socioeconómicos más bajos destinan una mayor proporción de sus ingresos a gastos de bolsillo para atender demandas en salud (Tabla 3). Sin embargo, dicho gasto varia muy poco entre los distintos servicios de salud, (Tabla 4). El gasto en medicamentos, es en promedio 52,4 \% (I.C $95 \% 38,7$ - 66,0), del gasto privado total de cada hogar, siendo los estratos más bajos los que poseen un porcentaje de gasto mayor en este rubro. El segundo lugar lo 
ocupa el gasto en hospitalización y cirugía con el $11 \%$ dentro del gasto total, encontrándose los estratos 4 y 6 (16\%) por encima de dicho promedio. El gasto privado en consulta médica solo abarca un $9 \%$ del gasto total; Se destaca el comportamiento del estrato 6 , el cual destina mayores recursos a la consulta médica que los estratos más bajos. Un aspecto a resaltar es el gasto en pago de seguros médicos privados, el que solo los estratos más altos están en capacidad de adquirir (13, 11 y $11 \%$ para los estratos 4, 5 y 6 respectivamente).

Tabla 4. Gasto privado de bolsillo (\%) en los distintos servicios de salud de los hogares. Cartagena de Indias 2004

\begin{tabular}{|c|c|c|c|c|}
\hline Variable & $\begin{array}{c}\text { Medica } \\
\text {-mentos }\end{array}$ & $\begin{array}{c}\text { Hospitaliza } \\
\text {-ción y cirugía }\end{array}$ & $\begin{array}{l}\text { Consulta } \\
\text { medica }\end{array}$ & $\begin{array}{l}\text { Laborato- } \\
\text { rio clínico }\end{array}$ \\
\hline \multicolumn{5}{|l|}{ Estrato Socioeconómico } \\
\hline Estrato SE (1) & 60 & 11 & 5 & 11 \\
\hline Estrato SE (2) & 67 & 11 & 10 & 5 \\
\hline Estrato SE (3) & 63 & 8 & 12 & 7 \\
\hline Estrato SE (4) & 48 & 16 & 10 & 6 \\
\hline Estrato SE (5) & 58 & 5 & 0 & 16 \\
\hline Estrato SE (6) & 47 & 16 & 11 & 0 \\
\hline \multicolumn{5}{|l|}{ Afiliación al SGSSS } \\
\hline Sin Afiliación & 65 & 6 & 6 & 7 \\
\hline Afil. Régimen Subsidiado & 54 & 11 & 8 & 13 \\
\hline Afil. Régimen Contributivo & 61 & 12 & 9 & 6 \\
\hline \multicolumn{5}{|l|}{ Escolaridad del jefe de hogar } \\
\hline Sin Escolaridad & 54 & 9 & 9 & 18 \\
\hline Primaria Incompleta & 56 & 17 & 8 & 13 \\
\hline Primaria completa & 63 & 15 & 6 & 10 \\
\hline Secundaria Incompleta & 71 & 4 & 6 & 9 \\
\hline Secundaria completa & 67 & 7 & 10 & 2 \\
\hline Técnico & 52 & 12 & 16 & 4 \\
\hline Universitario & 57 & 10 & 3 & 10 \\
\hline Postgrado & 50 & 16 & 8 & 0 \\
\hline \multicolumn{5}{|c|}{ Condición laboral del jefe de hogar } \\
\hline Desempleado & 44 & 3 & 11 & 25 \\
\hline Trabajador independiente & 58 & 9 & 10 & 8 \\
\hline Jubilado & 73 & 12 & 6 & 0 \\
\hline Trabajado dependiente & 61 & 12 & 6 & 5 \\
\hline
\end{tabular}

Escolaridad del jefe del hogar

La escolaridad del jefe del hogar estuvo asociada inversamente con el gasto privado como proporción del ingreso. Los porcentajes más altos se encuentran en niveles de escolaridad bajos ( $42 \%$ y $32 \%$ en primaria completa e incompleta 
respectivamente). $\mathrm{Al}$ hacer la diferencia de medias entre los grupos extremos según la escolaridad, los resultados muestran que existen diferencias significativas $(p=0,046$ y 0,002$)$ en la participación del gasto privado en salud como proporción del ingreso.

\section{Análisis Econométrico}

Los resultados de las estimaciones de los coeficientes del modelo de regresión Logístico binario, (Tabla 5) muestran los factores asociados al gasto de bolsillo catastrófico para el hogar.

Tabla 5. Regresion logistica binaria de la probabilidad de realizar un gasto de bolsillo en salud mayor $30 \%$ y $50 \%$ del ingreso total del hogar

\begin{tabular}{|c|c|c|c|c|c|c|}
\hline \multirow{3}{*}{ Variable } & \multicolumn{3}{|c|}{$30 \%$} & \multicolumn{3}{|c|}{$50 \%$} \\
\hline & \multirow{2}{*}{ OR } & \multicolumn{2}{|c|}{ I.C. $95,0 \%$} & \multirow{2}{*}{ OR } & \multicolumn{2}{|c|}{ I.C. $95,0 \%$} \\
\hline & & Inf. & Sup. & & Inf. & Sup. \\
\hline \multicolumn{7}{|l|}{ Estrato Socioeconómico* } \\
\hline \multicolumn{7}{|l|}{ Estrato SE (1) } \\
\hline Estrato SE (2) & 7,081 & 1,329 & 37,723 & 7,555 & 1,029 & 55,449 \\
\hline Estrato SE (3) & 7,559 & 1,533 & 37,269 & 8,378 & 1,231 & 57,014 \\
\hline Estrato SE (4) & 1,684 & 0,345 & 8,218 & 1,416 & 0,205 & 9,799 \\
\hline Estrato SE (5) & 1,723 & 0,329 & 9,034 & 2,982 & 0,431 & 20,619 \\
\hline Estrato SE (6) & 3,190 & 0,506 & 20,092 & 2,541 & 0,239 & 26,984 \\
\hline \multicolumn{7}{|l|}{ Afiliación al SGSSS* } \\
\hline \multicolumn{7}{|l|}{ Sin Afiliación } \\
\hline Afil. Régimen Subsidiado & 0,957 & 0,468 & 1,959 & 0,741 & 0,338 & 1,623 \\
\hline Afil. Régimen Contributivo & 0,366 & 0,150 & 0,893 & 0,311 & 0,115 & 0,842 \\
\hline \multicolumn{7}{|l|}{ Escolaridad del jefe de hogar* } \\
\hline \multicolumn{7}{|c|}{ Sin Escolaridad } \\
\hline Primaria Incompleta & 0,405 & 0,053 & 3,115 & 0,413 & 0,043 & 3,930 \\
\hline Primaria completa & 0,351 & 0,059 & 2,077 & 0,189 & 0,025 & 1,447 \\
\hline Secundaria Incompleta & 0,423 & 0,084 & 2,126 & 0,417 & 0,068 & 2,536 \\
\hline Secundaria completa & 0,346 & 0,070 & 1,703 & 0,257 & 0,042 & 1,553 \\
\hline Técnico & 0,278 & 0,058 & 1,329 & 0,310 & 0,053 & 1,796 \\
\hline Universitario & 0,300 & 0,063 & 1,430 & 0,243 & 0,041 & 1,431 \\
\hline Postgrado & 0,305 & 0,065 & 1,438 & 0,234 & 0,040 & 1,357 \\
\hline \multicolumn{7}{|l|}{$\begin{array}{l}\text { Condición laboral del jefe de } \\
\text { hogar* }\end{array}$} \\
\hline \multicolumn{7}{|l|}{ Desempleado } \\
\hline Trabajador independiente & 2,617 & 0,910 & 7,528 & 2,886 & 0,899 & 9,267 \\
\hline Jubilado & 1,493 & 0,759 & 2,937 & 1,721 & 0,794 & 3,731 \\
\hline Trabajado dependiente & 1,336 & 0,621 & 2,874 & 0,804 & 0,312 & 2,069 \\
\hline
\end{tabular}

Se obtuvo el estadístico Exp (B) que es la razón de ventajas u Odds Ratio (RV) para cada variable socioeconómica, teniendo en cuenta la primera categoría de la respectiva variable. Así, para el estrato socioeconómico todas 
las razones de ventaja halladas resultaron ser significativos aunque se observa que los estratos más altos presentan RV menores que los estratos más bajos para todos los niveles de gasto de bolsillo. En cuanto a la escolaridad del jefe del hogar, aunque las RV no resultan significativas se observa una tendencia a constituirse en factor protector contra el gasto catastrófico de los hogares. Lo mismo se puede decir acerca de la variable ocupación del jefe del hogar. Por otro lado, es más clara la relación con respecto a la variable afiliación en salud, en donde la afiliación al régimen contributivo es un factor protector en contra de incurrir en un gasto de bolsillo en salud del $30 \%$ y $50 \%$ del ingreso. Es de aclarar que se detectó de manera informal (a través de los gráficos de los residuos) heteroscedasticidad en los modelos donde la variable dependiente es gastar mas del $30 \%$ y $50 \%$ del ingreso.

\section{DISCUSIÓN}

El análisis de la composición de las fuentes de financiamiento y su comparación con el acceso diferencial a los servicios de salud por los distintos grupos de población permite extraer conclusiones sobre el grado de equidad financiera del sistema de salud (5). Y, la equidad en salud no puede dejar de ser una característica central de la justicia de los acuerdos sociales en general (9).

La equidad en la financiación de los sistemas de salud, según los igualitaristas, significa crear las condiciones para que cada persona, independientemente de su situación social y creencias personales, tenga acceso a los bienes y servicios que le permitan mantenerse sana, realizar sus aspiraciones y lograr un bienestar compatible con el desarrollo de la sociedad en que vive (10). Existe equidad vertical cuando se da tratamiento desigual para desiguales. En términos de financiación significa que aquellos con desigual capacidad de pago deben realizar pagos distintos al sistema. Así, aquellos que más recursos tienen contribuyen al sistema proporcionalmente a su nivel de renta. La equidad vertical de la financiación implica la redistribución final neta de la renta entre los miembros de una sociedad, de manera que los más ricos generarán recursos para los más pobres. La equidad vertical de una fuente de financiación equivale a la progresividad de la misma. Una fuente de financiación progresiva es aquélla en la que las contribuciones que realiza un individuo como proporción de su renta aumentan conforme aumenta su renta. La equidad vertical de la financiación de un sistema de salud se mide con índices de progresividad como el índice Kakwani a partir de encuestas de hogares (11). Este índice compara la renta que percibe cada decil de población 
con la contribución a la financiación de la salud que realiza. Si el sistema de financiación es progresivo, los más pobres pagan relativamente menos en comparación con su renta y los más ricos relativamente más.

En nuestro estudio, una familia que pertenece a los estrato 1 ó 2, tiene ingresos menores y muchas veces inciertos en comparación con estratos superiores. Sin embargo, el gasto de bolsillo en salud en proporción al ingreso del hogar, es mayor en dicha familia, lo cual muestra una clara situación de inequidad vertical en el financiamiento del sistema de salud. Esta situación de regresividad es acorde con la mostrada en el estudio desarrollado por Cendex (4), en donde, según la clasificación por grupos de ingresos de los hogares encuestados, se observa una disminución, en términos generales, de la participación del gasto de bolsillo en salud a medida que el ingreso promedio mensual de estos aumenta.

En Latinoamérica, la expansión de los mecanismos del mercado en los servicios de salud, ha hecho que una de las modalidades de financiamiento que se extendiera fuera la del cobro a los usuarios. En principio, el objetivo de este mecanismo consiste en focalizar el gasto público, cobrar por los servicios a las familias que tienen capacidad de pago y eximir o cobrar tarifas menores a las que no la tienen. La experiencia demuestra que esta modalidad de financiamiento se ha transformado en una barrera para el acceso a los servicios de salud y que su implementación ha generado problemas adicionales (5).

Por otro parte, la discusión del gasto privado en salud se enmarca en el debate entre dos corrientes ideológicas que pretenden solucionar problemas de desigualdad. Dichas corrientes contrastan la manera de utilizar recursos: Focalización vs Universalización. Por un lado se argumenta en contra de la focalización que provoca ciertas distorsiones como las limitaciones que tienen los grupos de población, en cuanto a poder político se refiere, que son objeto de programas selectivos lo que inclina la balanza hacia la universalización (12). De otro lado están los resultados regresivos que se pueden obtener en la universalización producto del aprovechamiento de subsidios por parte de grupos de población que no necesitan de estos.

La principal limitación del presente estudio radica en que debido a la aleatoriedad de la enfermedad es posible que se sobreestime la ponderación de esta en distintos grupos poblacionales, pues no se trata de un estudio longitudinal sino de un corte en algún momento del tiempo. 
El presente estudio ratifica lo observado en otras investigaciones $(3,8)$ que afirman que el gasto privado en salud (gasto de bolsillo), en términos absolutos, se incrementa en la medida que aumenta el ingreso de los hogares; Y, en términos relativos (como proporción del ingreso) se incrementa en la medida que se desciende en el estrato socioeconómico. De igual modo, los resultados del estudio brindan evidencia empírica del comportamiento que tienen los hogares cartageneros, según el estrato socioeconómico al cual pertenecen, en cuanto a la distribución de su gasto de bolsillo en salud. El estudio realizado por la CEPAL (13) encontró diferencias estadísticamente significativas (se presentó una disminución en el promedio) entre el gasto de bolsillo en medicamentos reportado por la Encuesta Nacional de Hogares en 1992 y el reportado por la misma encuesta en 1997. Los autores mencionan como probable explicación que la reforma a la salud tuvo un impacto importante en el gasto de bolsillo. Aún así, el presente estudio refleja que en la distribución del gasto de bolsillo en salud, el primer lugar de entre los rubros a que se destina el mismo, sigue siendo el dedicado a la adquisición de medicamentos, con mayor impacto para los estratos bajos los cuales incurren en proporciones mayores que los estratos más altos. Este panorama, enmarcado en un Tratado de Libre Comercio TLC- deja ver claro, quiénes se verían más afectados ante posibles alzas en los medicamentos, como consecuencia de la protección a patentes en este campo.

De otro lado, la naturaleza del estudio (corte transversal) permite observar el comportamiento de distintos agentes en un momento dado del tiempo, estimando el gasto privado trimestral en salud de los encuestados. A diferencia del estudio desarrollado por Castaño, que analizaron el gasto privado en salud por quintiles de ingreso, en esta investigación se analizó el gasto privado de bolsillo según estratos socioeconómicos, lo cual permite tener otra visión del problema. En dicho estudio se afirma que la situación de inequidad ha ido disminuyendo gracias a la reforma al sistema de salud, pero contrastándola con los resultados obtenidos en la presente investigación se concluye que aún falta equiparar las cargas para obtener mejores resultados. Esto se afirma al observar la situación que vivieron los hogares que gastaron en salud una proporción mayor a su ingreso disponible, al verse obligados a incurrir en una descapitalización que redujo o agotó sus ahorros, conduciéndolos a la venta de bienes o el endeudamiento lo que sustenta por qué la OMS considera que cuando los hogares incurren en un gasto en salud mayor del 30 \% de su ingreso, se está frente a una situación de catástrofe económica para el hogar. Suponemos entonces, que el gasto puede llegar a hacerse insostenible para hogares que pertenecen a un estrato de población con ingresos mensuales relativamente bajos. Estos resultados coinciden con los reportados en otro estudio anterior (14). 
La percepción acerca de que factores como la cobertura del seguro y la distribución desigual de la educación (sobre todo en la mujer que muchas veces suele ser la jefa del hogar) determinan la utilización de servicios de salud al tiempo que pueden llegar a convertirse en generadores de pobreza ha sido validada años atrás (15). Al observar la relación entre gasto promedio de bolsillo en salud y afiliación a la seguridad social, los no afiliados gastan en promedio casi el doble de los afiliados al régimen contributivo, lo cual implica pérdida de eficiencia en la financiación de la salud de los no afiliados, comparados con los que tienen alguna clase de afiliación, que otros autores han comentado (16). De la misma manera Cendex observa una baja participación en el gasto de bolsillo de las consultas médicas, indicando que el aseguramiento afecta el nivel de gasto (4).

Trabajos previos como el de Ramírez han establecido inequidades del gasto en salud vía capacidad de generación de ingresos de las personas cabeza de hogar (17). Nuestro estudio muestra evidencias de que esta afirmación es cierta, puesto que encuentra a los desempleados y trabajadores independientes destinando una mayor proporción de su ingreso a gastos de bolsillo en salud, comparados con aquellos que tienen un ingreso fijo, como son los trabajadores asalariados y los jubilados.

Aunque la Secretaría de Planeación Distrital de Cartagena afirma que la cobertura del SISBEN es del $100 \%$ (18), el estudio identificó que un $30 \%$ de los encuestados en los estratos 1 y 2 no se les había aplicado la encuesta SISBEN. Esto es relevante dado que en esta población se observa un comportamiento diferente al momento de buscar satisfacer sus necesidades de salud. Ellos, tienden a reemplazar la medicina tradicional por la automedicación y remedios caseros; $y$ en casos de urgencia, incurren en un mayor gasto privado (19).

Por último, en Cartagena, los hogares están reflejando un mayor empobrecimiento por ingresos y entre las razones que explican este comportamiento se encuentran: alta tasa de natalidad en los sectores populares, surgimiento de nuevos barrios de invasión caracterizados por una extrema pobreza y un mercado laboral estrecho e inestable, entre otras. Para el año 2004 la ciudad contaba con el 67 \% de la población por debajo de la línea de pobreza. Esta situación hace de Cartagena una ciudad con un grueso numero de habitantes en condiciones de vulnerabilidad para la cual son consistentes los datos anteriormente mostrados. El gasto de bolsillo, así medido, se 
constituye en un Proxy para evaluar la equidad en el financiamiento de los sistemas de salud (10). Así, dado que Cartagena de Indias se ha caracterizado por ser una de las ciudades con mayor desigualdad social en América Latina, se puede afirmar que la situación reflejada en el estudio, plantea una sinergia que potencia la exclusión (marginalidad social e inequidad en el acceso al financiamiento de los servicios de salud)œ

\section{REFERENCIAS}

1. Schultz T. 1962. Reflections on investment in man. Journal of Political Economy. 1962; 70: 101-109.

2. Torres AC, Knaul FM. Determinantes del Gasto de bolsillo en salud e implicaciones para el aseguramiento universal en México: 1992 - 2000. En: Caleidoscopio de la Salud. (2003) pp. 209-225. [Internet] Disponible en http:// www.funsalud.org.mx/casesalud/caleidoscopio/caleidoscopio.htm Consultado en junio 2005.

3. Grossman M. On the concept of health capital and the demand for health. Journal of Political Economy.1972; 80:223-255.

4. O’Meara BG, Ruiz GF, Amaya LJL. Impacto del aseguramiento sobre uso y gasto en salud en Colombia. 1a ed. Bogotá: Centro Editorial Javeriano; 2003.

5. Molina R, Pinto M, Henderson P, Viera C. Health spending and financing: the current situation and trends. Rev Panam Salud Publica, jul./ago. 2000; 8 (1-2):71-83.

6. Liebetrau AM. Measures of association. Newbury Park, CA: Sage Publications. Quantitative Applications in the Social Sciences Series No. 32; 1983.

7. Parker S, Wong R. Household income and health care expenditures in Mexico. Health Policy. 1997; 40: 237-255.

8. OMS The World Health Report 2000. Health systems: improving performance. Ginebra: OMS;2000.

9. Yepes LFJ. Gasto privado en salud. Colombia 1977-1980. Gac Med. Mex. 1984; 15(2):49-54.

10. Sen A. ¿Por quéla equidad en salud?. Rev Panam Salud Publica, May/June 2002; 11 (5)::302-309.

11. Wagstaff A, Van Doorslaer E., 1992. Equity in the finance and delivery of health care: Concepts and definitions. In: Wagstaff A, Van Doorlaer E, Rutten F (Ed.). Equity in the Finance and Delivery of Health Care An International Perspective. Oxford: Oxford University Press. pp. 85-103

12. Kakwani N C. Measurement of tax progressivity: An international comparison. Economic Journal. 1977; 87:71-80.

13. Sen A. Desarrollo y Libertad. Bogotá: Editorial Planeta; 2000. p. 169. 
14. Castaño R, Arbelaez J, Giedion U, Morales L. Evolución de la equidad en el sistema colombiano de salud. Santiago de Chile: CEPAL; 2000. p. 39.

15. Rocha A, Hernández P, Ruiz S, Ávila L, Marín T, Lazcano E. Gasto de hogares durante la hospitalización de menores derechohabientes, con diagnóstico de leucemia, en dos hospitales en México. Salud Pública de México. 2003; 45 (4): 291.

16. Organización Panamericana dela Salud. Crecimiento Económico y Pobreza. Informe del grupo de trabajo I de la comisión sobre Macroeconomía y Salud; 2003 pp. 51-53.

17. Baeza C, Cabezas M, Oyarzo C. El Gasto de bolsillo en salud en América Latina y el Caribe: Razones de eficiencia para la extensión de la protección social en salud. Organización Interamericana de Trabajo; 1999 p. 18.

18. Ramírez M, Cortés D, Gallego J. El Gasto en Salud de los Hogares Colombianos: Un análisis descriptivo. Serie Documentos Borradores de Investigación. Bogotá: Universidad del Rosario; Marzo 2002.

19. Secretaría de Planeación Distrital de Cartagena. Análisis Estadístico de la base de datos del SISBEN. Cartagena de Indias; Noviembre de 2003.

20. Vega R. Dilemas éticos contemporáneos en salud. El caso colombiano desde la perspectiva de la justicia social. Gerencia y Políticas de Salud. Septiembre 2002; 1(2):.49-65. 